\section{FRAILTY SYNDROME AND MORBID OBESITY}

Roger W. Byard

School of Medicine, The University of Adelaide, and Forensic Science SA, Adelaide, SA, Australia

Frailty syndrome is a relatively recently recognised entity that involves the concurrence of a number of specific clinical manifestations that include unintentional weight loss, decreased muscle mass (sarcopenia), reduced physical strength and activity, exhaustion and slow ambulation. Multiple systems are involved and it is an increasing problem as elderly populations expand in size, as it is strongly associated with increases in both morbidity and mortality. Although frailty syndrome has been discussed in the clinical literature for over a decade, it is not often identified in forensic situations. As there is a direct relationship between frailty syndrome and adverse health outcomes, the syndrome has clear medicolegal significance. Conversely, there has been such a striking increase in average body size in many countries over recent years that it has been referred to as an obesity 'epidemic'. This is associated with an increase in numbers of such cases in forensic mortuaries with significant practical difficulties in body handling and in identifying multiple, often complex, comorbidities. Of interest, morbid obesity and frailty syndrome involve interacting environmental, genetic and neuroendocrine factors, with systemic inflammatory responses and multiorgan dysfunction causing significant metabolic disturbances. More careful identification of both frailty syndrome and morbid obesity will give a better understanding of the incidence of extreme weight disturbances and their medicolegal consequences.

\section{DEFENDING CASES}

Philip Beh

Department of Pathology, The University of Hong Kong, Hong Kong

Defending cases is always a daunting task. This is ever more difficult when a case is sensational or involves vulnerable victims. It is not infrequent that writers demand that 'experts' must be impartial and must not be partisan. It is common practice that experts are required to make declarations of impartialness and of serving the truth and the court.

In the adversarial system, legal practitioners have a long history of experience of manoeuvering and staying within the rules but they are by nature 'partisan' and advocates for their side. The expert medical witness is expected to be different and does often try their very best to be impartial. There is however one inherent flaw, the experts can only be as impartial as the information provided. It is often argued that experts must point out 'important missing information'. Anyone who has tried to do this will very quickly find themselves hypothesising into the 'land of absurdity'.

This talk will highlight with examples some of the scenarios and how best one can ensure that justice is served. At the end of the day, it is important that 'experts' remain professionally indifferent and assist the court to understand the evidence available.

\title{
Clinical Forensic Medicine
}

\section{DNA DECONTAMINATION PROTOCOLS}

\author{
$\underline{\text { Sanjeev Gaya }}$ \\ Victorian Institute of Forensic Medicine, Southbank, Vic, \\ Australia
}

\begin{abstract}
Aim: To determine the effectiveness of a cleaning protocol at removing DNA material from selected surfaces and items in a sexual assault examination room after deliberate contamination. A secondary aim is to determine the extent of background DNA by analysis of control samples.

Background: The new Multi-Disciplinary Centre (MDC) in Dandenong, Victoria, includes two adjacent purpose-built examination suites dedicated for sexual assault examinations. The effectiveness of current cleaning protocols at removing DNA material from the sexual assault examination suites at the MDC remains untested.

Method: Standardised and diluted single-donor blood samples, which when dried were not visible to the naked eye, were smeared onto pre-determined surfaces and items in a previously cleaned and sealed sexual assault examination room. Control samples were taken from the pre-determined sites before and after contamination using certified DNA free swabs dampened with DNA free water. A forensic clean was requested in the normal fashion and a trained cleaner, who was blinded to the deliberate contamination, cleaned the suite in accordance with established protocols after a sexual
\end{abstract}

assault examination at the MDC. Within 12-24 hours of cleaning, samples were obtained from the previously contaminated sites. All samples, including positive and negative controls, were forwarded to the laboratory for nuclear DNA analysis.

The current cleaning protocols and the results will be presented. Discussion: Potential contamination from the facility in which forensic samples are obtained can have significant impact on their forensic integrity and their admissibility in and reliance by the courts. A cleaning regimen that reliably removes sources of biological material from an examination room between examinations reduces, but does not eliminate, the chance of cross-contamination.

\section{REDUCING RISKS AND IMPROVING HEALTH OUTCOMES FOR PRISONERS IN WATCH HOUSES: DO EMERGENCY DEPARTMENT NURSES IN THE CUSTODIAL SETTING MAKE A DIFFERENCE?}

$\underline{\text { C. Lincoln }}^{1}, \underline{\text { J. Crilly }}^{2,3}$, P. Scuffham ${ }^{2}$, J. Timms ${ }^{3}$, K. Becker ${ }^{4}$, N. van Buuren ${ }^{1}$, A. Fisher ${ }^{3}$, D. Murphy ${ }^{5}$, D. Green ${ }^{2,3}$ ${ }^{1}$ Clinical Forensic Medicine Unit, Queensland Department of Health, ${ }^{2}$ Menzies Health Institute Queensland, Griffith University, ${ }^{3}$ Department of Emergency Medicine, Gold Coast Hospital and Health Service, ${ }^{4}$ Southport Watchhouse, Queensland Police Service, and ${ }^{5}$ Queensland Ambulance Service, Qld, Australia 